\title{
Effects of three months treatment with sertraline on intraocular pressure and cup-to-disc ratio in patients with anxiety disorders/mixed anxiety and depressive disorder/major depressive disorder and without underlying eye disease
}

\author{
Samaneh Farnia ${ }^{1}$, Hamid Bazyari², Hanieh Ahmadi ${ }^{3}$, Nasim Tabrizi $^{4}$, Athena sharifi_razavi ${ }^{4}$, \\ Narjes Hendouei $5^{*}$
}

${ }^{1}$ Psychiatry and Behavioral Sciences Research Center, Addiction Institute and Department of Psychiatry, Mazandaran University of Medical Sciences, Sari, Iran

2Student Research Committee, Faculty of Pharmacy, Mazandaran University of Medical Sciences, Sari, Iran ${ }^{3}$ Department of ophthalmology, School of medicine, Mazandaran University of Medical Sciences, Sari, Iran ${ }^{4}$ Department of Neurology, School of medicine,Mazandaran University of Medical Sciences, Sari, Iran

${ }^{5}$ Department of Pharmacotherapy, Faculty of Pharmacy and Psychiatry and Behavioral Sciences Research Center, Addiction Institute

, Mazandaran University of Medical Sciences, Sari, Iran

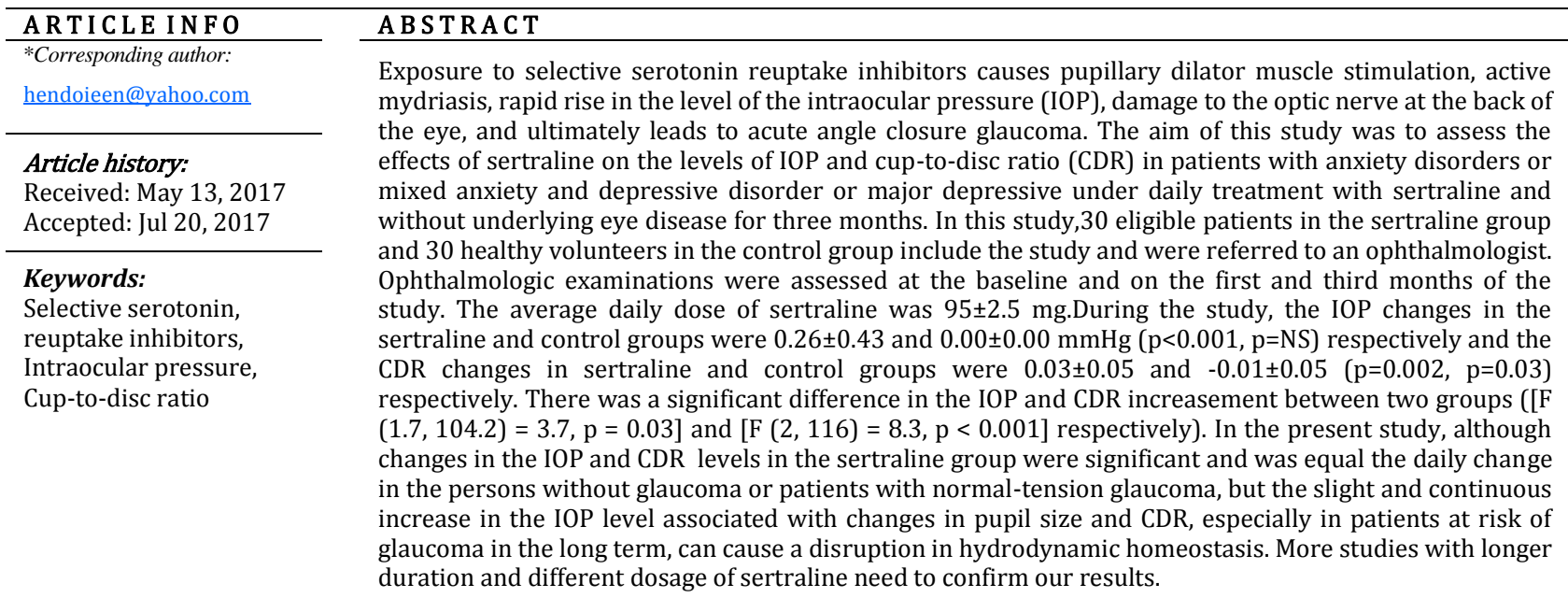

Citation: Pharm Biomed Res 2017;3(3):31-37.

\section{Introduction}

Sertraline is an antidepressant drug belonging to the selective serotonin reuptake inhibitors (SSRIs) group. It is widely used in treating patients with anxiety, depression, or other types of heterogenic mood disorders(1). In the last two decades, mood disorders have developed to an impressive extent and the prescription of SSRIs has increased because of its safety and tolerable profile in comparison with other antidepressant drugs(2-5).Ocular complications of SSRIs are rarely detected and can be accompanied by dangerous consequences(6).Short-term SSRI exposure causes pupillary dilator muscle stimulation, active mydriasis, a sudden blockage of the drainage of aqueous humor fluid out of the eyes, a rapid rise in the intraocular pressure (IOP) level, damage to the optic nerve at the back of the eye,ultimately leading to acute angle closure glaucoma(AACG)(7). AACG is an ocular emergency and requires immediate treatment. Rapid diagnosis, immediate intervention, and referral can have profound effects on patient outcome and morbidity (8). Studies revealed that the probability of glaucoma-especially AACG-is higher with SSRIs that have a stronger norepinephrine and serotonin reuptake inhibitor (17). Among SSRIs, sertraline has the highest potency in serotonin reuptake inhibition and is the most potent SSRI in the inhibition of norepinephrine reuptake after paroxetine (14).Yet, several cases of acute glaucoma were reported in patients treated with 
paroxetine(9-13),citalopram(3,14), escitalopram (15), and sertraline (7). Recently, Lochhead has reported five cases of optic neuropathy in patients treated with paroxetine for 14 years(one case), citalopram for three and 10 years(two cases), fluoxetine for five years(one case), sertraline for six months and citalopram for six months(one case)(16).In most of the cases mentioned above, ocular complications of SSRIs occurred in women more than 50 years old and with underlying medical illness, and treated with drugs other than antidepressants(17). On August 12, 2016, Health Canada published a safety review titled "Antidepressants-Assessing the Potential Risk of Serious Eye Disorder (Angle-Closure Glaucoma),"based on a case-control study using a Taiwanese healthcare insurance database over an 11year period (2000-2011). The safety review focused on the 23 drugs-antidepressant medications-available in Canada.The results showed a 5.8-fold increased risk of AACG at the beginning of the use of SSRIs. However,theresults were limited to Taiwan's population, known as a high-risk group;the visual effects of medication use other than antidepressants were notassessed,which may be a mainprobable confounder(6).Studies on the effects of the long-term use of SSRIs on the level of intraocular pressure are very limited(17).The findings of the retrospective cohort study by Chen et al. in 2015 showed that more than a year'sconsumption of SSRIs was not accompanied with an increased risk of primary openangle glaucoma (POAG) or primary angle-closure glaucoma (PACG) in patients with depression(8).In contrast, the recent study revealed that the probability of glaucoma in patients younger than 65 years, treated with SSRIs at doses equal to or higher thanone defined daily dose for one year or more, is significantly higher than that of the control group(17).

According to an analysis of the related literatures,the dataon the association between chronic consumption of SSRIs and changes in IOP level and optic nerve head function in patients without underlying eye and noneye diseases are limited.Their results are also associated with controversies(7, 9-15, 18, 19).Now, it is unclear whether the risk of ocular complications of SSRIs is limited to high-risk patients, such as a family history of the condition, migraines, high blood pressure, obesity, old age, female sex, Eskimo or Asian ethnicity, or whether itoccurs in all cases $(14,16)$.To the best of our knowledge,no prospective studyhasbeen reported so far to assess the trend of intraocular pressure andcup-to-disc ratio (CDR) in patients treated with SSRIs and without underlying eye and non-eye diseases.This study is the first one to assess the effect of sertraline on the level of IOP and CDRin patients with anxiety disorders or mixed anxiety and depressive disorder or major depressive disorder aged between 20 and 40 years old with no underlying eye diseaseswho have beenunder dailytreatment withsertralinefor three months.

\section{Materials and Method}

Study Design and Participants

This longitudinal descriptive study was conducted in Baghban Clinic affilated Mazandaran University of Medical Sciences, located in Sari in the north of Iran, between May and September 2015. It was approved by the Ethics Committee of Mazandaran University of Medical Sciences (registration ID: IR.MAZUMS.REC.95.1459). First, the study was explained to the eligible patients, and if they agreed to participate in the study, an informed consent was obtained.

The inclusion criteria were patients aged between 20 and 40 years old with anxiety disorders or mixed anxiety and depressive disorder or major depressive disorder, according to DSM-IV-TR criteria(20) and IOP between 12-16 mm $\mathrm{Hg}$ and did not receive any kinds of SSRI in the past six months.

Subjects were excluded if they took illicit drugs (opiates, amphetamines, cannabis, etc.), alcohol, cigarettes, any kinds of topical or systematic drugs influencing IOP, such as beta blocker, alpha $1^{-}$ adrenergic receptor agonist, calcium channel blocker and other types of antihypertensive drugs, prostaglandin compound, tricyclic antidepressants, monoamine oxidase inhibitors, serotoninnorepinephrine reuptake inhibitors,and/or anticholinergic drugs in the past six months. Patients who needed to increase the sertraline dose (more than $100 \mathrm{mg}$ daily)orto add another medication during the study period,and the patients who tooksystemic glucocorticoids intermittently in two weeks before starting the study, or had a continuous therapy with systemic glucocorticoids one month prior to the study were also excluded. Also, subjects were omitted if they had glaucoma or any other kind of underlying ophthalmologic disorder, positive family history for glaucoma, eye laceration, and laser eye surgery in the past three months or an eye trauma in the past six months, loss of vision in one eye, contact lensand diabetes, kidney,liver and cardiovascular diseases, and cerebrovascular accidents. Thirty age- and gendermatched healthy volunteers with IOP between 12 and $16 \mathrm{~mm} \mathrm{Hg}$ were included as the control group from general population. The control group did not receive any kinds of SSRI in the last six months and did not have ocular and non-ocular diseases.

\section{Sample size}

During the periodfrom May to September 2015, 80 participants were registered in the trial; 37 patients in thesertraline group and 40 healthy volunteers in the control group met the inclusion criteria. The sample 
size-considering 95\% confidence, $80 \%$ power, $20 \%$ drop out-was calculatedbased onthe study of Costagliola et al.(19),and wasdefined as a $20 \%$ or 8 $\mathrm{mmHg}$ increase in IOP levels from baseline to study end point; there were 30 patients in the sertraline group and 30 healthy volunteers as the control one.

\section{Procedures}

Patients who met the inclusion criteria were included in the study and receivedsertraline (made by Sobhan Co. Iran), with an initial dose of $25 \mathrm{mg} /$ day for three days; if tolerated, the dose was increased to $50 \mathrm{mg}$ daily. Patients were re-evaluated after four weeks and the sertraline dosage was increased to $100 \mathrm{mg} /$ day, if required.

The measurement of the degree of adherence to medications was carried out by a comparison of weekly tablet counts with participant reports of medication intake in order to estimate the proportion of dispensed medication that was actually ingested.

\section{Outcome}

In this study, IOP, retina, visual acuity, anterior chamber angle, and automated perimetry were assessedby an ophthalmologist at the baseline and on the first and third months of the study. IOP was measured by using Goldmann's tonometry method, on the base of Haag-Strei(21), examining the retinaincluding CDR - by the slit lamp method, using a 90 diopter lens(22).The visual acuity was measured by a Snellen chart (23).Gonioscopy(24)was also conducted to assess the anterior chamber angle and automated perimetry(25). The ophthalmologist wasnot informed of the both groups.

Intraocular pressure was measured at 4-6 p.m. in the sitting position. All participants were asked not to use products containing caffeine 24 hours before the measurement ofIOP. IOP was measured twice, and if the difference was more than $2 \mathrm{mmHg}$, it was measured for the third time. Then, the IOP mean was calculated and analyzed.

For each participant, a set of variables was collected, including demographics (age and gender), visual acuity, the presence of anterior chamber angle, levels of IOP and CDRand underlying psychiatric disorder.

\section{Safety}

All patients underwent a complete physical examination at the beginning of the trial and each subsequent visit. Serum creatinine, blood urea nitrogen (BUN), alanine transaminase (ALT), aspartate aminotransferase (AST), fasting blood glucose level, low-density lipoprotein cholesterol (LDL-C) level, serum total cholesterol level, high-density lipoprotein cholesterol level (HDL-C), thyroid function tests, hematocrit, hemoglobin, platelet, and white blood cell (WBC) count were measured at the baseline to rule out underlying medical conditions. Through open-ended questioning as well as a complete checklist of side effects, adverse effects were recorded at each session. We requested the patients to immediately inform us about any unexpected unfavorable symptom during the period of the trial.

\section{Statistical analysis}

All data were assessed for normality by the "one sample Kolmogorov-Smirnov" test. Qualitative variables were recorded in accordance with frequency and percentage and quantitative variables in terms of Mean \pm SD(Standard Deviation). To compare continuous variables in two groups, we used the independent sample t-test, and to compare for categorical variables, either Chi-Square or Fisher's exact test. Repeated measurement analysis was conducted for serial comparisons of IOP and CDR and comparisons between groups at different times of treatment; pairwise comparison was applied by Scheffé. All statistical analyses were conducted using the software SPSS Version 21 (SPSS Inc., Chicago, IL, USA) and P values of less than 0.05 were considered significant.

\section{Results}

Thirty-seven eligible patients in the sertraline group and 40 healthy volunteers in the control group were referred to an ophthalmologist at the beginning of the study. Six healthy volunteers at the end of the first month in the control group, two patients in the sertraline group, and four healthy volunteers at the end of the third month in the control group did not cooperate in ophthalmic examinations. The sertraline dosage was increased at the end of the first month by the lack of improvement of the symptoms in four patients and one at the end of the third month in the sertraline group. Finally, 60 patients completed the study: 30 of them received sertraline and 30 were healthy volunteers. There was no significant difference between the groups in baseline demographic and clinical measurements. The average daily dose of sertraline was $95 \pm 2.5 \mathrm{mg}$ daily in sertraline groups.Baseline demographics and clinical characteristics were shown in Table 1.Baseline mean IOP levelwas $12.1 \pm 1.1(95 \% \mathrm{CI}=11.7-12.5)(\mathrm{mmHg})$ and $12.05 \pm 0.56(95 \% \mathrm{CI}=11.8-12.2)(\mathrm{mmHg})$ for sertraline and control groups respectively $(\mathrm{p}=0.6)$. During the study, the mean IOP level increased by $0.1 \pm 0.5$ (95\% $\mathrm{CI}=-0.03-0.3) \mathrm{mmHg}$ at the end of the first month and $0.26 \pm 0.43(95 \% \mathrm{CI}=0.1-0.4) \mathrm{mmHg}$ at the end of the third month compared with the baseline in the sertraline group. The increase at the end of the third month compared with the baseline was statistically significant in the sertraline group $(\mathrm{p}<0.001)$. The mean IOP changes were $-0.016 \pm 0.3 \quad(95 \% \mathrm{CI}=-0.15-$ $0.11) \mathrm{mmHg}$ and $0.00 \pm 0.00 \mathrm{mmHg}$ at the end of the first and third months compared to the baseline in the 
control group respectively ( $\mathrm{p}=0.8, \mathrm{p}=\mathrm{NS}$ respectively). General linear model repeated measure showed a significant effect for time $\times$ intervention interaction between both groups for IOP increasement at the end of the third[F $(1.7,104.2)=3.7, p=0.03]$. Post hoc analyses using the Scheffé post hoc criterion for significance indicated that the increase in mean IOP levels at the end of the third month, compared to the baseline, had a significant difference between groups $(p=0.004)$ (Table 2).
Baseline mean CDR level was 0.19 \pm 0.06 (95\%CI=0.17$0.2)$ and $0.21 \pm 0.05(0.19-0.24)$ in sertraline and control groupsrespectively $(\mathrm{p}=0.1)$.During the study, the mean CDR level increased significantly at the end of the first and the third months compared with the baseline in sertraline group $(\mathrm{p}<0.001, \mathrm{p}=0.002$ respectively). In the control group, the mean CDR level decreased insignificantly at the end of the first month, $-0.01, \pm 0.04$, (95\%CI $=-0.02-0.007)$ and third month, $-0.01, \pm 0.05$, (95\%CI $=-0.03-0.009)$ compared to the baseline $(\mathrm{p}=0.2$, $\mathrm{p}=0.3$ respectively).

Table 1 Baseline demographic and clinical measurements in all patients

\begin{tabular}{lccc}
\hline Characteristics & $\begin{array}{c}\text { Sertraline group }(\mathrm{n}=30) \\
\text { Mean } \pm \text { SD }(95 \% \mathrm{CI})\end{array}$ & $\begin{array}{c}\text { Control group }(\mathrm{n}=30) \\
\text { Mean } \pm \text { SD }(95 \% \mathrm{CI})\end{array}$ & $P$ value \\
\hline Age $(\mathrm{y})$ & $33.8 \pm 4.2(32.2-35.4)$ & $32.4 \pm 4.2(30.4-33.6)$ & 0.1 \\
\hline Gender (F/M), N(\%) & $25(83.3) / 5(16.7)$ & $20(66.7) / 10(33.3)$ & 0.1 \\
\hline Visual acuity & $1.8 \pm 0.6(1.5-2)$ & $2.1 \pm 0.7(1.8-2.3)$ & 0.08 \\
\hline Diagnosis, N(\%) & $23(76.7)$ & - & - \\
Anxiety disorder & $4(13.3)$ & & \\
Depressive disorder & $3(10)$ & Open & - \\
Mixed anxiety depressive disorder & Open & $12.05 \pm 0.56(11.8-12.2)$ & 0.6 \\
\hline Anterior chamber angle & $12.1 \pm 1.1(11.7-12.5)$ & 0.1 \\
\hline IOP (mmHg) & $0.19 \pm 0.06(0.17-0.2)$ & $0.21 \pm 0.05(0.19-0.24)$ & 0.1 \\
\hline CDR & & & \\
\hline Deta
\end{tabular}

Data are presented as Mean \pm SD $(95 \% \mathrm{CI})$, or Number $(\%)$, where applicable. SD: standard deviation; CI: confidence interval; IOP: Intraocular pressure; CDR: Cup to disc ratio.

Table 2 Intraocular pressure level variations during treatment in all patients

\begin{tabular}{lccc}
\hline IOP $(\mathbf{m m H g})$ & $\begin{array}{c}\text { Sertraline group (n=30) } \\
\text { Mean } \pm \text { SD (95\%CI) }\end{array}$ & $\begin{array}{c}\text { Control group (n=30) } \\
\text { Mean } \pm \text { SD (95\%CI) }\end{array}$ & $P$ value \\
\hline Baseline & $12.1 \pm 1.1(11.7-12.5)$ & $12.05 \pm 0.56(11.8-12.2)$ & 0.6 \\
\hline At the end of the first month & $12.3 \pm 1.1(11.9-12.7)$ & $12.03 \pm 0.62(11.7-12.2)$ & 0.2 \\
\hline $\begin{array}{l}\text { Difference between the end of } \\
\text { the first month and baseline }\end{array}$ & $0.1 \pm 0.5(-0.03-0.3)$ & $-0.016 \pm 0.3(-0.15-0.11)$ & 0.1 \\
\hline $\begin{array}{l}\text { At the end of the third month } \\
\text { Difference between the end of } \\
\text { the third month and baseline }\end{array}$ & $0.26 \pm 0.43(0.1-0.4)$ & $12.05 \pm 0.56(11.8-12.2)$ & $0.00 \pm 0.00$ \\
\hline $\begin{array}{l}\text { Difference between the end of } \\
\text { the third month and the end of } \\
\text { the first month }\end{array}$ & $0.1 \pm 0.4(-0.06-0.2)$ & $0.01 \pm 0.3(-0.15-0.11)$ & 0.4 \\
\hline
\end{tabular}

Data are presented as Mean \pm SD (95\%CI), or Number (\%), where applicable. SD: standard deviation; CI: confidence interval; IOP: Intraocular pressure. 
In data analysis, there was a significant difference between the two groups in the mean CDR changes at the end of the first and third months compared with baseline $(p=0.002, p=0.001$ respectively) (Table 3 ). General linear model repeated measure showed a significant effect for time $\times$ intervention interaction between both groups for CDR increasement at the end of the third $[\mathrm{F}(2,116)=8.3, \mathrm{p}<0.001]$.

No serious adverse event occurred. In the sertraline group, dizziness, decreased appetite, and insomnia occurred in two, one, and two patients respectively during the first week, which were resolved with continued treatmentin the second week.

\section{Discussion}

To the best of our knowledge, this study is the first one to assess the effect of sertraline on the levels of IOP and CDR in patients with anxiety disorders or mixed anxiety and depressive disorder or major depressive disorder aged between 20 and 40 years under daily treatment with sertraline and without underlying eye and non-eye diseases for three months. The results of the present study showed that the IOP level increased significantlyin the sertraline group and was unchanged in the control group during the study. Also, the mean CDR level increased significantly at the end of the first month and remained unchanged till the end of the study in the sertraline group, but the mean CDR level decreased in the control group.

Costagliola et al.(19)studied the effects of a single dose fluoxetine, $20 \mathrm{mg}$ /day on IOP in 20 depressed patients without any underlying disease. IOP was measured at baseline and on anhourly basis up to 12 hours. The results showed a significant increase in IOP from 90 minutes to eight hours after the administration of fluoxetine with a maximum in the fourth hour.
After the eighth hour, a significant increase in IOP was reported compared to the control group. In this study, the patients' mean IOP at baseline $(16 \mathrm{~mm} \mathrm{Hg})$ and age range (33-47 years old) were higher than in the present study $(12 \mathrm{mmHg}$ and $30-35$ years oldrespectively), and $75 \%$ of patients were females in their study. Bonomi revealed that the possibility of acute glaucoma attacks in old age and among womenis higher due to a less peripheral anterior chamber depth compared with youths and men(26). In a retrospective cohort study (7),the probable incidence of primary angle-closure glaucoma and primary open-angle glaucoma in depressed patients above 20 years, treated by SSRIs without preexisting glaucoma for one year, was investigated. The mean follow-up periods were 5.5 and 5.7 years for the SSRI and the control grouprespectively. Their results showed that there was no significant difference between patients receiving SSRIs and control group in the incidence of primary angle-closure glaucoma. The study was conducted retrospectively and the data gathered from the Taiwan Insurance System, so there were some factors that affected the results such as the uncertainty associated with the use of recorded SSRIs for each patient and the lack of clinical data in glaucoma diagnosis such as the IOP level, corneal thickness, visual acuity, and evaluation of optic nerve and underlying diseases.Also, this study only evaluated depressed patients, while SSRIs are prescribed in a variety of psychiatric and nonpsychiatric diseases.

In our work, the IOP increased during the study, and the difference was meaningful compared with the baseline at the end of the third month in the sertraline group. In our study, the cup-to-disc ratio increased in the sertraline group, but it decreased in the control group at the end of the first month.

Table 3 CDR variations during treatment in all patients

\begin{tabular}{lccc}
\hline CDR & $\begin{array}{c}\text { Sertraline group }(\mathrm{n}=30) \\
\text { Mean } \pm \text { SD }(95 \% \mathrm{CI})\end{array}$ & $\begin{array}{c}\text { Control group (n=30) } \\
\text { Mean } \pm \text { SD (95\%CI) }\end{array}$ & $P$ value \\
\hline Baseline & $0.19 \pm 0.06(0.17-0.2)$ & $0.21 \pm 0.05(0.19-0.24)$ & 0.1 \\
\hline At the end of the first month & $0.2 \pm 0.08(0.19-0.25)$ & $0.2 \pm 0.06(0.18-0.23)$ & 0.4 \\
\hline $\begin{array}{l}\text { Difference between the end of } \\
\text { the first month and baseline }\end{array}$ & $0.02 \pm 0.04(0.01-0.04)$ & $-0.01 \pm 0.04(-0.02-0.007)$ & 0.1 \\
\hline At the end of the third month & $0.2 \pm 0.08(0.2-0.26)$ & $0.2 \pm 0.05(0.18-0.22)$ & 0.001 \\
\hline $\begin{array}{l}\text { Difference between the end of } \\
\text { the third month and baseline }\end{array}$ & $0.03 \pm 0.05(0.01-0.05)$ & $-0.01 \pm 0.05(-0.03-0.009)$ & 0.3 \\
\hline $\begin{array}{l}\text { Difference between the end of } \\
\text { the third month and the end of } \\
\text { the first month }\end{array}$ & $0.01 \pm 0.04(-0.007-0.02)$ & $-0.001 \pm 0.05(-0.02-0.01)$ & \\
\hline
\end{tabular}

Data are presented as Mean \pm SD $(95 \% \mathrm{CI})$, or Number $(\%)$, where applicable. SD: standard deviation; CI: confidence interval; CDR: Cup to disc ratio. 
The cup-to-disc ratio was fixed at the end of the study in both groups compared with the end of the first month. There was a meaningful difference between two groups in the cup-to-disc ratio change at the end of the first month and the third month. Although a change in the IOP level can equal the daily change in the IOP level in persons without glaucoma or patients with normaltension glaucoma(5),the change in the IOP level associated with changes in pupil size and cup-to-disc ratio, especially in patients at risk of glaucoma, can cause a disruption in hydrodynamic homeostasis(27). Also, Hayer et al. showed that the increase in plasma serotonin levels can disturb blood supply in the optic nerve head, leading to ischemic optic neuropathy,independent of IOP levels(27). It seems that during chronic treatment with SSRIs, the incidence of several temporary spam in the optic nerve head vessels causes hemodynamic changes and, eventually, ischemic optic neuropathy (28).A major populationbased survey based on theInternational Society Geographical and Epidemiological Ophthalmology(ISGE0), which studied the prevalence and types of glaucoma in Yazd province in central of Iran, showed that the overall prevalence of glaucoma was $4.4 \%$ and the prevalence of open-angle glaucoma and angle-closure glaucoma were $1.7 \%$ and $0.4 \%$ respectively(5).Also, the prevalence of normal-tension glaucoma was relatively high $(1.5 \%)$. According to these results, some factors except IOP-such as cup-todisc ratio(27)and serum sodium levels $(29,30)$ - play a role in glaucomatous optic neuropathy in the Iranian race.

This study has some limitations, which should be pointed out. In this study, we evaluated the intraocular pressure changes induced by SSRIs in individuals aged 20-40 years.According to the Australian Adverse Drug Reaction Advisory Committee (ADRAC), drug-induced glaucoma is more likely to develop in people aged 3270 years.Also, population-based, nested case-control study in 2017 reported that the occurrence of glaucoma in patients treated with SSRIs and younger than 65 years is more than in older people(17).However, old age is one of the main and well-known risk factors forglaucoma,and its role in drug-induced glaucomacannot be ignored(28).

Another limitation is the sertraline dose. In the present study, the mean sertraline dose was $95 \pm 2.5 \mathrm{mg}$ daily. Previous studies have revealed $(2,17,27,29,31)$ that the occurrence of this effect depends on the drug plasma level and its metabolites,occurring at a mean daily dose of SSRIs exceeding $20 \mathrm{mg}(31)$ or $\geq 1$ defined daily dose(17).

In this study, we evaluated the effect of sertraline on the level of IOP and CDR in patients with anxiety disorders, mixed anxiety and depressive disorder, major depressive disorder. But SSRIs are prescribed in a variety of psychiatric and non-psychiatric diseases,which may be act as a factor influencing the level of IOP and CDR.

Although structured clinical interviews were conducted for all participants at the baseline by a psychiatrist, we did not examine the personality characteristics of both groups. According to the Bubella study, Type A behavior pattern has a significant impact on the daily fluctuations of the IOP and the occurrence of openangle glaucoma in people without ocular disease (32). In addition, there was no quantitativeassessment of the level of participants' anxiety and depression severity at the beginning and the duration of study. The results of Zhou et al.'s study showed that anxiety and depression severity are directly linked toself-reported visual function disturbance(33).

\section{Conclusion}

In the present study, change in levels of the IOP and CDR in the sertraline group were significant compared to control group during the study. Although change in the IOP levels, in our study, was equal the daily change in the IOP level in persons without glaucoma or patients with normal-tension glaucoma(5), but the slight and continuous increase in the IOP level associated with changes in pupil size and CDR , especially in patients at risk of glaucoma in the long term, can cause a disruption in hydrodynamic homeostasis(27). More studies need to confirm our results We recommend that various studies be designed with different doses of sertraline and other types of SSRIs, participants' anxiety and depression severity levels and personality characteristics assessments, and longer periods of treatment with more patients, especially patients with risk factors of glaucoma, such as old age, female, positive family history of glaucoma, mid to high hyperopia and cataract, and the ones who receive other drugs or have underlying diseases, which affect the process of metabolism of SSRIs or disrupt the elimination of SSRIs. Also,we suggest that studies be designed to address the impact of SSRIs on the level of IOP and CDR in medical or psychiatric illnesses other than those covered in this study.

\section{Conflict of Interest}

No conflicts of interest have been declared.

\section{Source of Funding}

This research was financially supported by Mazandaran University of Medical Sciences, Sari, Mazandaran, Iran.

\section{References}

1. Şener EC, Kıratlı H. Presumed sertraline maculopathy. Acta Ophthalmologica Scandinavica. 2001;79:428-30.

2. Costagliola C, Parmeggiani F, Semeraro F, Sebastiani A. Selective serotonin reuptake inhibitors: a review of its effects on intraocular pressure. Curr neuropharmacol 2008;6:293-310. 
3. Croos R, Thirumalai S, Hassan S, Davis JD. Citalopram associated with acute angle-closure glaucoma: case report. BMC ophthalmol 2005;5:23.

4. Suri F, Yazdani S, Elahi E. Glaucoma in Iran and contributions of studies in Iran to the understanding of the etiology of glaucoma. J ophthalmic vis res 2015;10:68-76.

5. Pakravan M, Yazdani S, Javadi MA, Amini H, Behroozi Z, Ziaei H, et al. A population-based survey of the prevalence andtypes of glaucoma in central Iran: the Yazd Eye Study. Ophthalmology 2013;120:1977-84.

6. Ho HY, Kam K-WA, Young AL, Chan LK, Yu EC-S. Acute angle closure glaucoma after sertraline. Gen hosp psychiatry 2013;35:575.e1-2.

7. Chen HY, Lin CL, Kao CH. Long-Term Use of Selective Serotonin Reuptake Inhibitors and Risk of Glaucoma in Depression Patients. Medicine (Baltimore) 2015;94:e2041.

8. Browning AC, Reck AC, Chisholm LH, Nischal KK. Acute angle closure glaucoma presenting in a young patient after administration of paroxetine. Eye 2000;14:406-8.

9. Bennett $\mathrm{H}$, Wyllie A. Paroxetine and acute angle-closure glaucoma. Eye 1999;13:691-2.

10. Levy J, Tessler Z, Klemperer I, Shneck M, Lifshitz T. Late bilateral acuteangle-closure glaucoma after administration of paroxetine in a patient with plateau iris configuration. Can J Ophthalmol 2004;39:780-1.

11. Kirwan J, Subak-Sharpe I, Teimory M. Bilateral acute angleclosure glaucoma after administration of paroxetine. $\mathrm{Br} \mathrm{J}$ Ophthalmol 1997;81:252.

12. Eke T, Bates A. Acute angle closure glaucoma associated with paroxetine. BMJ 1997; 314:1387.

13. Massaoutis P, Goh D, Foster P. Bilateral symptomatic angle closure associated with a regular dose of citalopram, an SSRI antidepressant. Br J Ophthalmol 2007;91:1086-7.

14. Zelefsky JR, Fine HF, Rubinstein VJ, Hsu IS, Finger PT. Escitalopram-induceduveal effusions and bilateral angle closure glaucoma. Am J Ophthalmol. 2006;141:1144-7.

15. Lochhead J. SSRI-associated optic neuropathy. Eye 2015;29:1233-5

16. Chen HY, Lin CL, Lai S-W, Kao CH. Association of selective serotonin reuptake inhibitor use and acute angle-closure glaucoma. J Clin Psychiatry 2016;77:e692-6.

17. Mediero A, Alarma-Estrany P, Pintor J. New treatments for ocular hypertension. Auton Neurosci 2009;147:14-9.

18. Costagliola C, Mastropasqua L ,Capone D, Verolino $\mathrm{M}$, Ciancaglini M, Pisanti N. Effect of fluoxetine on intraocular pressure in the rabbit. Exp Eye Res 2000;70:551-5.

19. Costagliola C, Mastropasqua L, Steardo L, Testa N. Fluoxetine oral administration increases intraocular pressure. BrJ Ophthalmol 1996;80:678.
20. Costagliola C, Parmeggiani F, Sebastiani A. SSRIs and intraocular pressure modifications. CNS Drugs 2004;18:475-84

21. Association AP. Diagnostic and statistical manual of mental disorders (DSM).4th ed-TR. Washington, DC: American psychiatric association. 1994:143-7.

22. Ozcura F, Yildirim N, Sahin A, Colak E. Comparison of Goldmann applanation tonometry, rebound tonometry and dynamic contour tonometry in normal and glaucomatous eyes .Int J Ophthalmol 2015;8:299-304.

23. Hoffmann EM, Zangwill LM, Crowston JG, Weinreb RN. Optic disk size and glaucoma. Surv Ophthalmol 2007;52:32-49.

24. Universale CO. Visual acuity measurement standard. Italian Journal. 1984

25. Faschinger C, Hommer A. How to Perform Gonioscopy. Gonioscopy: Springer; 2012.p. 5-10.

26. Khuu SK, Kalloniatis M. Standard Automated Perimetry: Determining Spatial Summation and Its Effect on Contrast Sensitivity Across the Visual FieldSpatialSummation Effect on Visual Sensitivity. Invest Ophthalmol Vis Sci 2015 ;56:3565-76.

27. Caiado RR, Badaró E, Kasahara N. Intraocular pressure fluctuation in healthy and glaucomatous eyes: a comparative analysis between diurnal curves in supine and sitting positions and the water drinking test. Arq Bras Oftalmol 2014;77:288-92.

28. Bengtsson B, Leske MC, Hyman L, Heijl A, Group EMGT. Fluctuation of intraocular pressure and glaucoma progression in the early manifest glaucoma trial. Ophthalmol 2007;114:2059

29. Hashemi H, Kashi A, Fotouhi A, Mohammad K. Distribution of intraocular pressure in healthy Iranian individuals: the Tehran Eye Study. Br J Ophthalmol 2005;89:652-7.

30. BonomiL, Marchini G, Marraffa M, Bernardi P, De Franco I, Perfetti S, et al. Prevalence of glaucoma and intraocular pressure distribution in a defined population: the EgnaNeumarkt Study. Ophthalmol 1998;105:209-15.

31. Bogner B, Runge C, Strohmaier C, Trost A, Tockner B, Kiel JW, et al. The Effect of Vasopressin on Ciliary Blood Flow and Aqueous FlowEffect of Vasopressin on CilBF and AqF. Invest Ophthalmol Vis Sci 2014;55:396-403.

32. Bubella RM, Bubella DM, Cillino S. Type A behavior pattern: is it a risk factor for open-angle chronic glaucoma? J Glaucoma 2014;23:199-201

33. Zhou C, Qian S, Wu P, Qiu C. Anxiety and depression in Chinese patients with glaucoma: sociodemographic, clinical, and selfreported correlates. J. Psychosom Res 2013;75:75-82. 